\title{
Ionization Rates for Atmospheric and Ionospheric Breakdown
}

\author{
K. Papadopoulos, ${ }^{1,2,3}$ G. Milikh, ${ }^{1,3}$ A. Gurevich, ${ }^{4}$ \\ A. Drobot, ${ }^{5}$ and R. Shanny ${ }^{6}$
}

\begin{abstract}
The differences in the ionization rates for RF induced atmospheric breakdown between the analytic formula given by Gurevich et al. [1978] and recent numerical simulations Short et al., 1991; Tsang et al., [1991] were examined and clarified. An updated analytic formula for the ionization rate of air, which includes corrections due to kinetic effects of the electron distribution function, was derived. The formula is valid for arbitrary ratios of $\mathrm{RF}$ to electron-neutral collision frequency.
\end{abstract}

\section{IONIZATION RATES}

Theoretical and experimental studies of air breakdown in the presence of strong microwave fields were performed as early as the late 1950s and early 1960s [McDonald, 1966]. These studies emphasized breakdown under controlled laboratory conditions mostly in microwave cavities. Kroll and Watson [1972] extended the microwave breakdown computations to optical and infrared laser frequencies including the effects of multiphoton absorption. Interest on the subject has been recently renewed, since the development of microwave sources and phased arrays provides the technological capability to create plasma clouds in the atmosphere and ionosphere, using focused ground-based installations, which transmit short high-power pulses of microwaves [Gurevich, 1980]. These plasma clouds can be used as artificially ionized mirrors (AIM) for communications and radar applications [Borisov et al., 1986; Armstrong et al., 1989; Duncan and Milikh, 1989; Short et al., 1990; Tsang et al., 1991]. Recent studies [Papadopoulos, 1990] indicate that optical and other emissions from such plasma clouds can be used to diagnose remotely minority and toxic species in the stratosphere.

A critical quantity in assessing the RF power requirements for pulsed breakdown experiments is an accurate knowledge of the ionization rate $\nu_{1}$ and its dependence on the local RF electric field $E_{o}$, frequency $\omega$, and ambient air neutral density $\mathrm{N}_{\mathrm{m}}$ or pressure $P$. Empirical formulae for $\nu_{\mathrm{i}}$ have been presented by several authors [Ali, 1981; Lupan, 1976; Mayhan and DeVore, 1968]. These formulae are, however, valid for limited ranges of parameters, often contradictory and without solid physical justification [Short et al., 1990; Borisov et al., 1986]. The consistent approach, which is valid for any meaning of the radio wave frequency is associated with the kinetic theory of breakdown.

An analytic theory for the ionization rate was developed by Gurevich et al. [1978] (hereafter referred to as I). An important contribution of the paper was the recognition that $\nu_{i}$ is a function of two dimensionless parameters $E_{o} / E_{k}$ and $\omega / \nu_{k}$. The value of the effective collision frequency $\nu_{k}$ is only a function of the ambient gas density given by

\footnotetext{
${ }^{1}$ Department of Astronomy, University of Maryland, College Park.

${ }^{2}$ Also at University of Maryland, Department of Physics, College Park.

3 Also at ARCO Power Technologies, Incorporated, Washington, D. C.

${ }^{4}$ Lebedev Institute of Physics, Moscow, Russia.

${ }^{5}$ Science Application International Corporation, McLean, Virginia.

${ }^{6}$ ARCO Power Technologies, Incorporated, Washington, D. C.
}

Copyright 1993 by the American Geophysical Union.

Paper number 93JA00795.

0148-0227/93/93JA-00795\$05.00

$$
\begin{aligned}
\nu_{\mathrm{s}} & =1.6 \times 10^{-7}\left(\frac{\mathrm{N}_{\mathrm{m}}}{\mathrm{cm}^{3}}\right) \mathrm{s}^{-1}= \\
& =6 \times 10^{9}\left(\frac{\mathrm{P}}{\text { Torr }}\right) \mathrm{s}^{-1}
\end{aligned}
$$

The value of the characteristic field $E_{k}$ depends in addition to the value of $\mathrm{N}_{\mathrm{m}}$, on the frequency $\omega$ in the form of $\omega / \nu_{k}$. It is defined as

$$
\begin{aligned}
\mathrm{E}_{\mathrm{k}}= & 32\left(\frac{\mathrm{N}_{\mathrm{m}}}{2.7 \times 10^{19} \mathrm{~cm}^{-3}}\right) \sqrt{1+\frac{\omega^{2}}{\nu_{\mathrm{k}}^{2}}}= \\
& 32\left(\frac{P}{760 \text { Torr }}\right) \sqrt{1+\frac{\omega^{2}}{\nu_{k}^{2}}} \frac{\mathrm{kV}}{\mathrm{cm}}
\end{aligned}
$$

The ionization rate was given in $I$ in the form

$$
\frac{\nu_{\mathrm{i}}}{\nu_{\mathrm{m}}}=\mathrm{C}_{1} \mathrm{~F}\left(\frac{\mathrm{E}_{\mathrm{o}}}{\mathrm{E}_{\mathrm{k}}}\right)\left(\frac{\mathrm{E}_{\mathrm{o}}}{\mathrm{E}_{\mathrm{k}}}\right)^{2} \exp \left[-C_{2}\left(\frac{\mathrm{E}_{\mathrm{k}}}{\mathrm{E}_{\mathrm{o}}}\right)\right]
$$

In (3), $\nu_{m}$ is given by

$$
\nu_{\mathrm{m}}=7.6 \times 10^{-13}\left(\frac{\mathrm{N}_{\mathrm{m}}}{\mathrm{cm}^{3}}\right) \mathrm{s}^{-1}
$$

and the function $F(x)$ is defined as

$$
F(x) \equiv \frac{1+6.3 \exp (-2.6 / x)}{1.5}
$$

The two terms of $F(x)$ reflect the differences in the ionization properties of $\mathrm{N}_{2}$ and $\mathrm{O}_{2}$, the dominant atmospheric components taken as $80 \%$ and $20 \%$ of the neutral density. For values of $\mathrm{E}_{\mathrm{o}} / \mathrm{E}_{\mathrm{k}} \approx 1-5$ relevant to ionospheric breakdown the factor $F$ varies between 1 and 3 .

The parameters $C_{1}$ and $C_{2}$ in (3) are parametrizations of the integrals of inelastic cross sections over the energy. They depend on matching the electron distribution function in energy regions where different inelastic losses dominate (see I for details). In the general case, $C_{1}$ and $C_{2}$ are functions of the wave frequency. However, in $I$ it was assumed that $C_{1}$ and $C_{2}$ are constant with values

$$
\mathrm{C}_{1}=5.5 \times \mathrm{e}^{6}, \mathrm{C}_{2}=6
$$

By using (5) and (6), the ionization rate $\nu_{i}^{\mathrm{I}}$ may be extrapolated:

$$
\frac{\nu_{\mathrm{i}}^{\mathrm{I}}}{\nu_{\mathrm{m}}}=\left(5.5 \times \mathrm{e}^{6}\right) \mathrm{F}\left(\frac{\mathrm{E}_{\mathrm{o}}}{\mathrm{E}_{\mathrm{k}}}\right)\left(\frac{\mathrm{E}_{\mathrm{o}}}{\mathrm{E}_{\mathrm{k}}}\right)^{2} \exp \left[-6 \frac{\mathrm{E}_{\mathrm{o}}}{\mathrm{E}_{\mathrm{k}}}\right]
$$


This equation can be represented in the form

$$
\frac{\nu_{\mathrm{i}}^{\mathrm{I}}}{\nu_{\mathrm{m}}}=5.5 \mathrm{~F}\left(\frac{\mathrm{E}_{\mathrm{o}}}{\mathrm{E}_{\mathrm{k}}}\right)\left(\frac{\mathrm{E}_{\mathrm{o}}}{\mathrm{E}_{\mathrm{k}}}\right)^{2} \exp \left\{-6\left(\frac{\mathrm{E}_{\circ}}{\mathrm{E}_{\mathrm{k}}}-1\right)\right\}
$$

A set of computer simulations of ionospheric breakdown performed recently [Short et al., 1990; Tsang et al., 1991] (hereafter referred to II), while confirming the general dependence of $\nu_{i}$ on $E_{o} / E_{k}$ and $\omega / \nu_{k}$, indicated that (7) significantly overestimates the value of $\nu_{i}$ and consequently underestimates the required power for pulsed breakdown. In the high-frequency regime $\left(\omega>\nu_{k}\right)$ the simulation results were consistent with the analytic expression [Papadopoulos, 1990], (hereafter referred to as III):

$$
\nu_{\mathrm{i}}^{\mathrm{III}} / \nu_{\mathrm{m}}=1.2 \times 10^{3}\left(\frac{\tilde{\epsilon}}{\mathrm{eV}}\right) \exp \left(-\sqrt{\frac{2 \mathrm{eV}}{\tilde{\epsilon}}}\right)
$$

where $\tilde{\epsilon}$ is the electron quiver energy in electron volts defined as

$$
\tilde{\epsilon}=\frac{1}{2} m \frac{\mathrm{e}^{2} \mathrm{E}_{\mathrm{o}}^{2}}{\mathrm{~m}^{2} \omega^{2}}
$$

In the $\omega \gg \nu_{\mathrm{k}}$ range the value of $\tilde{\epsilon}$ corresponding to $E_{k}$ is $\tilde{\epsilon}_{k}=.08 \mathrm{eV}$.

A comparison of (7a) and (7b) with (8a) and (8b) along with the results of the numerical simulations [Short et al., 1990; Tsang et al., 1991] for $\omega \gg \nu_{k}$ is shown in Figure 1 (see also Table 1). Equation (8) gives results consistent with the simulation up to $\mathrm{E}_{\mathrm{o}} / \mathrm{E}_{\mathrm{k}} \approx 5$. It can be seen also that (7) overestimates the ionization rate by more than an order of magnitude over the entire range. This statement is backed up by the comparison of the simulation results with the collection of experimental data adopted from Sharfman and Morita [1964]. When the values of $E_{e} / p, \mathrm{~V} / \mathrm{cm}$ Torr used in the mentioned paper, where transformed into the units of $E_{o} / E_{k}$. we assumed that $\omega / \nu_{\mathbf{k}}<1$.

This overestimate has important implications in the microwave power requirements in performing atmospheric breakdown experiments as can be seen from Table 2. Planning based on (7) requires a value of electric field smaller by a factor of 1.5 -1.8 in the breakdown regign than the numerical results. This is translated into an underestimate of the microwave power by a factor of 3 .

In the next section we discuss briefly the causes of the discrepancy and determine the values of the parameters $C_{1}$ and $C_{2}$ as a function of $\omega / \nu_{\mathrm{k}}$, which are required to reconcile the results of reference I with these of reference II.

\section{UPDATED IONIZATION RATES: KINETIC CORRECTIONS}

The difference in the ionization frequency between reference I and II can be attributed to several factors. First, it is attributed to differences in the inelastic collision cross sections that were used. Reference I used cross sections adapted from Frost and Phelps [1962], and Hake and Phelps [1967], while reference II used updated values based on recent measurements [Ali, 1981]. Second, the approximation used by the analytic theory in reference I, equivalent to the Born approximation, is not sufficiently accurate over the entire range. Finally, the parameters $C_{1}$ and $C_{2}$ in (3) are not constant, as was assumed in reference I, but are functions of $\omega / \nu_{\mathrm{k}}$.

In the remainder of this section we utilize the self-similar form for the ionization rate as a function of $E_{\circ} / E_{k}$ and $\omega / \nu_{k}$ given

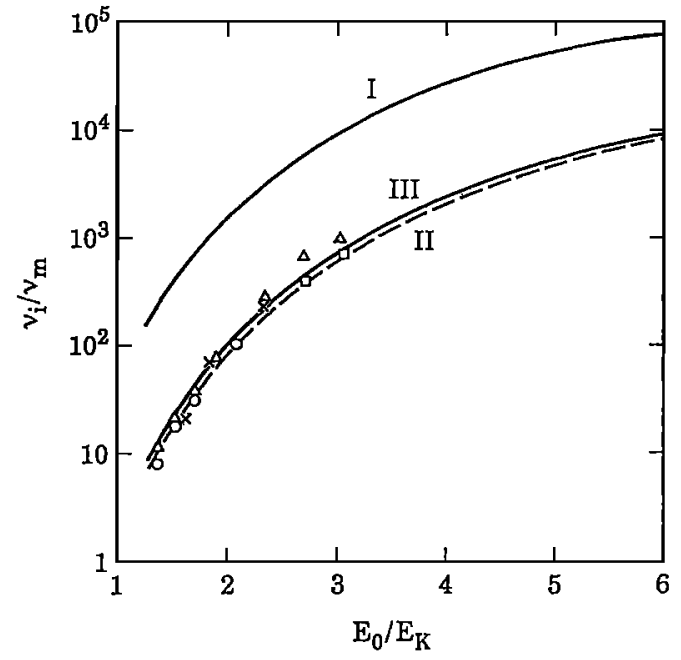

Fig. 1. The ionization frequency $\nu_{1}$ normalized over $\nu_{\mathrm{m}}$ as obtained in high frequency regime $\left(\omega>\nu_{k}\right)$ for different amplitude of the electric field of microwave. Curves I and III correspond to the analytical theory referred as I and III, while curve II corresponds to computer simulation, referred as II. Shown by open circles, open triangles, crosses, and boxes is the collection of the different experimental data adopted from Sharfman and Morita [1964, Figure 1].

TABLE 1. Ionization Frequency $\nu_{\mathrm{i}}$ Normalized Over $\nu_{\mathrm{m}}$, Which Is Obtained for Different Amplitude of the Electric Field of Microwave $\left(\omega \gg \nu_{k}\right)$

\begin{tabular}{llllllll}
\hline \multicolumn{7}{c}{$E_{0}\left(\frac{k V}{m}\right) \times 10^{4}$} \\
& 0.61 & 0.87 & 1.1 & 1.48 & 1.5 & 1.9 & 2.4 \\
$\mathrm{E}_{\mathrm{o}} / \mathrm{E}_{\mathrm{k}}$ & 1.32 & 1.86 & 2.28 & 3.19 & 3.23 & 4.07 & 5.13 \\
$\frac{\nu_{l}^{I}}{\nu_{m}} / 10^{3}$ & 0.2 & 1.0 & 2.7 & 10.7 & 11.3 & 26.0 & 56.3 \\
$\frac{\nu_{l}^{I I}}{\nu_{m}}$ & 8.1 & 72 & 210 & 930 & 970 & 2,250 & 4,650 \\
$\frac{\nu_{l}^{I I I}}{\nu_{m}}$ & 7.9 & 66 & 190 & 810 & 860 & 2,040 & 4,490 \\
$\frac{\nu_{l}^{(9)}}{\nu_{m}^{0}}$ & 7.2 & 60 & 180 & 840 & 880 & 2,210 & 5,060 \\
\hline
\end{tabular}

I, III conespond to the analytical theory refered as I, III II - to the computer simulation. Frequencies obtained by formula (9) are indicated as $\nu_{i}{ }^{(9)}$.

by (3), in conjunction with the computer code used in reference II, to derive a new accurate analytic formula which describes the ionization rate for the entire range of $\omega / \nu_{k}$. To accomplish this, we assume $\nu_{i}$ in the form

$$
\begin{gathered}
\frac{\nu_{\mathrm{i}}}{\nu_{\mathrm{m}}}=\mathrm{F}\left(\frac{\mathrm{E}_{\mathrm{o}}}{\mathrm{E}_{\mathrm{k}}}\right)\left(\frac{\mathrm{E}_{\mathrm{o}}}{\mathrm{E}_{\mathrm{k}}}\right)^{2} \times \\
\mathrm{C}_{1}{ }^{\prime}\left(\frac{\omega}{\nu_{\mathrm{k}}}\right) \exp \left[-4.7\left(\mathrm{C}_{2}^{\prime}\left(\frac{\omega}{\nu_{\mathrm{k}}}\right) \frac{\mathrm{E}_{\mathrm{k}}}{\mathrm{E}_{\mathrm{o}}}-1\right)\right]
\end{gathered}
$$

The coefficient 4.7 appears in the exponent of (9) instead of coefficient 6 given in (7), to account for the use of updated inelastic cross sections. The parameters $C_{1}^{\prime}\left(\omega / \nu_{k}\right)$ and $C_{2}^{\prime}\left(\omega / \nu_{k}\right)$ will be determined numerically. They are essentially kinetic coefficients. To determine these coefficients, a series of numerical simulations were performed, to compute the ionization rate as a function of $x=E_{o} / E_{k}$ for fixed values of $\omega / \nu_{k}$. For each value of $\omega / \nu_{k}$ the values of $\mathrm{C}_{1}^{\prime}$ and $\mathrm{C}_{2}^{\prime}$ were determined from the relationship 
TABLE 2. Electric Field Required to Produce Corresponding Ioniza -tion Frequency $\nu_{\mathrm{i}} / \nu_{\mathrm{m}}$ in High-Frequency Regime $\left(\omega>>\nu_{\mathbf{k}}\right)$

\begin{tabular}{lccc}
\hline \multicolumn{3}{c}{$\nu_{3} / \nu_{m}$} \\
\hline & 30 & 300 & 1000 \\
$\frac{E_{0}^{I}}{E_{k}}$ & 1.1 & 1.44 & 1.83 \\
$\frac{E_{0}^{I I}}{E_{k}}$ & 1.65 & 2.5 & 3.25 \\
\hline
\end{tabular}

$E_{o}^{I} / E_{k}$ and $E_{o}^{I I} / E_{k}$ are calculatëd by the different methods described in references I and II correspondingly.

$$
\mathrm{f}=\ln \left[\frac{\nu_{\mathrm{i}}(\mathrm{x})}{\nu_{\mathrm{m}}} \frac{1}{\mathrm{x}^{2} \mathrm{~F}(\mathrm{x})}\right]=4.7+\ln \mathrm{C}_{1}^{\prime}-\frac{C_{2}^{\prime}}{\mathrm{x}}
$$

shown in Figure 2.

The linear part of this function defines the constant $C_{2}^{\prime}\left(\omega / \nu_{k}\right)$. while the cutoff value $f_{0}$ at the ordinate axis is $4.7+\ln C_{1}^{\prime}$, i.e. $C_{1}^{\prime}=\exp \left(f_{o}-4.7\right)$. Figure 3 shows the functions $C_{1}^{\prime}$ and $C_{2}^{\prime}$. It can be seen that under $\omega<<\nu_{\mathrm{k}}$, both $C_{1}^{\prime}$ and $C_{2}^{\prime}$ tend to 1.0. In the opposite case $\omega>\nu_{\mathrm{k}}, \mathrm{C}_{1}^{\prime} \rightarrow 1.5, \mathrm{C}_{2}^{\prime} \rightarrow 1.1$.

As can be seen from Table 1 , in the high-frequency regime $\omega>\nu_{\mathrm{k}}$ (9) with $\mathrm{C}_{1}^{\prime}=1.5$ and $\mathrm{C}_{2}^{\prime}=1.1$ reduces to the analytic formula of reference III, given by (8) and is, of course, consistent with the results of the computer simulation.

In general, the analytic formula given by (9) with the corresponding kinetic coefficients $C_{1}^{\prime}\left(\omega / \nu_{k}\right), C_{2}^{\prime}\left(\omega / \nu_{k}\right)$ presents values of the ionization frequency $\nu_{i}$ with an the accuracy better than $10 \%$ for the amplitude of electric field close to the threshold $\left(\mathrm{E}_{\mathrm{o}} / \mathrm{E}_{\mathrm{k}} \leq 3\right)$. If the electric field increases up to $5 E_{k}$ the accuracy of ionization frequency obtained by formula (9) would be no better than $20-25 \%$. For the fields above $5 E_{k}$ the simple kinetic approach developed in references I-III can be no longer applicable, since under such conditions the mean electron energy tends to the ionization energy which makes the ionization process nonstationary. The mean source of energy losses becomes associated with the ionization and heating of the newly born electrons. It changes the form of the Fokker-Plank equation and effects the electron distribution function. This requires different analysis [Borisov et al., 1986].

\section{IONIZATION THRESHOLD}

It is customary in the literature to refer to a breakdown power or electric field threshold. The definition of the threshold depends critically on the dominant losses occurring in the particular experiment and pulse length of the microwaves. The lowest possible threshold occurs if we assume asymptotically long irradiation time and neglect convective or diffusive losses. In this case a unique definition of the threshold is possible as the power density (or electric field) at which the electron losses due to dissociative attachment of $\mathrm{O}_{2}$ equal the ionization rate. The threshold electric field $E_{t h}$ as defined above can be found by equating the ionization rate to the attachment rate. The values of $E_{\text {th }}$ were measured in dc field [see Kroll and Watson, 1972]. For the high-frequency microwaves the $E_{t h}$ was obtained in reference II. Using computer simulation of the same type as in reference II, we can find that

$$
\mathrm{E}_{\mathrm{th}}\left(\frac{\omega}{\nu_{\mathrm{k}}}\right)=\mathrm{C}_{\mathrm{th}}^{\prime}\left(\frac{\omega}{\nu_{\mathrm{k}}}\right) \mathrm{E}_{\mathrm{k}}
$$

where the coefficient $\mathrm{C}_{\mathrm{th}}^{\prime}$ is 1.0 when $\omega / \nu_{\mathbf{k}}<<1$ and becomes equal to 1.6 when $\omega / \nu_{\mathbf{k}}>>1$.

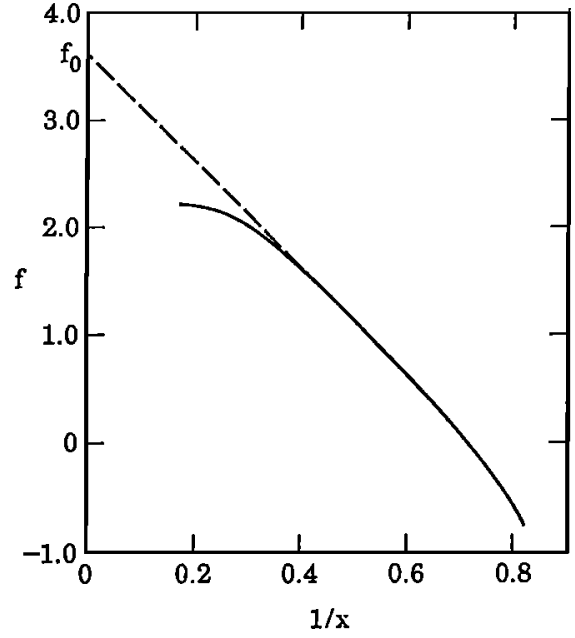

Fig. 2. Ilustration of the method of determination of kinetic coefficients, which is based on the relationship (10).

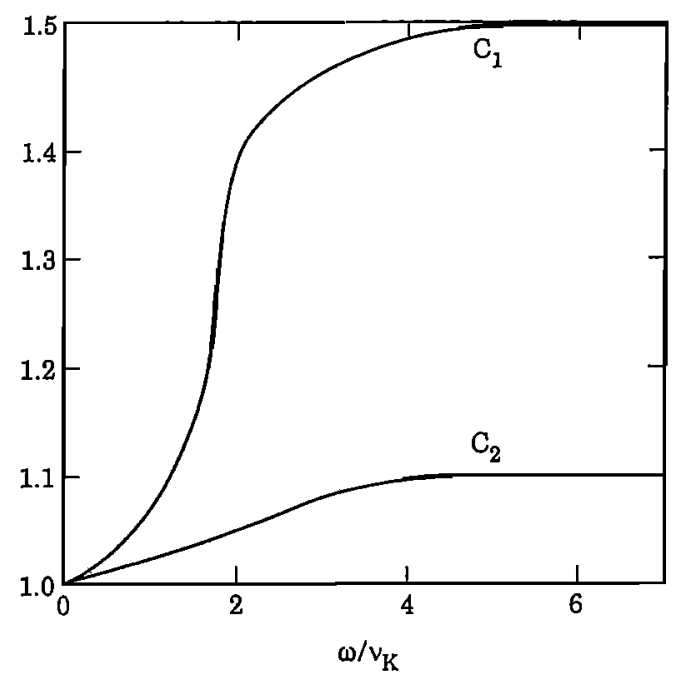

Fig. 3. The kinetic coefficients $C_{1}^{\prime}$ and $C_{2}^{\prime}$ as a functions of $\omega / \nu_{\mathrm{k}}$.

\section{SUMMARY AND CONCLUSIONS}

The reasons for the differences in the ionization rates between reference I and references II and III were discussed. Using the selfsimilar functional form for the ionization rate derived in reference I in conjunction with computer simulations, an analytic form for the ionization rate as a function of the RF frequency and power was derived, which is valid for the entire collisionality range (that is, $\omega / \nu_{\mathrm{k}} \lessgtr 1$ ) and for $\mathrm{E}_{\circ} / \mathrm{E}_{\mathrm{k}}<5$.

Acknowledgments. We acknowledge discussions with W. Ali, R. Short, T. Wallace and $\mathrm{K}$. Tsang. The work has been supported by the Office of Naval Research grant N0001490K201 (GM and AG) and Air Force Phillips Laboratory contract F19628-89-C—0174 (KP, AD, RS).

The Editor thanks M.S. Zahniser and an other referee for their assistance in evaluating this paper.

\section{REFERENCES}

Ali, A. W., Excitation and ionization cross sections for electron beam and microwave energy deposition in air, NRL Memo. Rep. 4598, 1981.

Armstrong, W. T., R. Roussel-Dupre, R. Alvarez, et al., Single and repetitive pulse AIL laboratory experiments, paper presented at the: 
Workshop on Artificial Ionized Layers in the Atmosphere, URSI, Kiev, USSR, October 9-13, 1989.

Borisov, N. D., A. V. Gurevich, and G. M. Milikh, Artificial Ionization of the Atmosphere, (in Russian), Academy of Science, Moscow, 1986.

Duncan, L. M., and G. M. Milikh, Arecibo AIL Design - Theory and Experiment, paper presented at the: Workshop on Artificial Ionized Layers in the Atmosphere, URSI, Kiev, USSR, October 9-13, 1989.

Frost, L. S., and A. V. Phelps, Rotational Excitation and Momentum Transfer Cross Sections for Electrons in $\mathbf{H}_{2}$ and $\mathbf{N}_{2}$ from Transport Coefficients, Phys. Rev. , 127, 1621-1631, 1962.

Gurevich, A. V., An ionized layer in a gas (in the atmosphere), Sov. Phys. Usp., Engl. Transl., 23(12), 862-865, 1980.

Gurevich A. V., G. M. Milikh, and I. S. Shlyuger, Changing Ionization of the Lower Ionosphere Due to High-Power Radio Waves, Radiophys. Quantum Electron., 20, 1230-1239, 1978.

Hake, R. D. Jr., A. V. Phelps, Momentun-transfer and inelastic-collision cross sections for electrons in $\mathrm{O}_{2}, \mathrm{CO}$, and $\mathrm{CO}_{2}$, Phys. Rev., 158, 70-84, 1967.

Kroll, N., and K. M. Watson, Theoretical study of ionization of air by intense laser pulses, Phys. Rev., 5, 1883-1905, 1972.

Lupan, Yu., A., Refined theory for an RF discharge in air, Sov. Phys. Tech. Phys., 21, 1367-1370, 1976.

Mayhan, J. T., and R. V. DeVore, Interaction of intense fields with heated air, J. Appl. Phys., 39, 5745-5753, 1968.

McDonald, A., Microwave Breakdown in Gases, John Wiley, New York,
1966.

Papadopoulos, K., Recent developments in ionospheric heating radio waves, in Topical Conference on Research Trends in Nonlinear and Relativistic Effects in Plasmas, California Space Institute, La Jolla, 1990.

Sharfman, W., and T. Morita, Focused Microwave Technique for Measurement of the Ionization Rate and Collision Frequency, J. Appl. Phys., 35, 2016-2020, 1964.

Short, R., D., et al., Physics Studies in Artificial Ionospheric Mirror (AIM) Related Phenomena, Tech. Rep. GL-TR-90-0038, Geophysics Lab., Hansom Air Force Base, Mass., 1990.

Tsang, K., K. Papadopoulos, A. Drobot, P. Vitello, T. Wallace, and R. Shanny, RF Ionization of the Lower Ionosphere, Radio Sci., 26(5), 1345-1360, 1991.

A. Drobot, Space Application International Corporation, McLean, VA 22102.

A. Gurevich, Lebedev Institute of Physics, Moscow V-333 Russia.

G. Milikh and K. Papadopoulos, Astronomy Department, University of Maryland, College Park, MD 20742.

R. Shanny, ARCO Power Technologies, Incorporated, Washington, DC 20037.

(Received March 27, 1992; revised December 1, 1992; accepted March 1, 1993.) 